\title{
Relationship of cardiovascular disease risk factors and noncoding RNAs with hypertension: a case-control study
}

Shiying Chen ${ }^{1 \dagger}$, Rong Chen ${ }^{1 \dagger}$, Tingxing Zhang ${ }^{2}$, Shaowei Lin ${ }^{1}$, Zhou Chen ${ }^{3}$, Bi Zhao ${ }^{4}$, Huangyuan $\mathrm{Li}^{*}$ and Siying $\mathrm{Wu}^{1 *}$ (iD

\begin{abstract}
Background: The present study sought to explore the relationship of common cardiovascular disease risk factors and noncoding RNAs with essential hypertension (EH).

Methods: A total of $402 \mathrm{EH}$ patients and 402 gender- and age-frequency matched healthy controls were enrolled in this study. Each participant received a questionnaire survey, physical examination and laboratory tests. Quantitative real-time polymerase chain reaction (qPCR) was performed to assess relative expression levels of six noncoding RNAs (NR_027032, NR_034083, NR_104181, miR-126, miR-143 and miR-145) in peripheral blood leucocytes. Multiple logistic regression analysis was used to estimate the risk of having EH between hypertensive and non-hypertensive patients.

Results: Analysis showed that participants with anxiety, high body mass index, abdominal obesity and family history of hypertension had higher risk for $\mathrm{EH}$, whereas those with bland diet and occupational physical activities had lower risk for EH. GPCR assays showed that NR_027032 $(P=0.015)$ and NR_034083 $(P=0.004)$ were significantly reduced in EH patients compared with controls, whereas NR_104181 $(P=0.007)$, miR-143 $(P=0.005)$ and miR-145 $(P=0.015)$ were significantly elevated.

After controlling the cardiovascular risk factors, multivariate analysis showed that lower expression levels of NR_034083 and higher expression levels of NR_104181 and miR-143 were risk factors for EH.

Conclusions: $\mathrm{EH}$ is a result of environmental and epigenetic factors. Strikingly, NR_034083, NR_104181 and miR-143 may be correlated with the risk for EH development; therefore, epigenetic markers could be used to measure hypertension levels to help elucidate the pathogenesis of $\mathrm{EH}$.
\end{abstract}

Keywords: Long noncoding RNAs, MicroRNAs, Cardiovascular disease risk factors, Essential hypertension

\section{Background}

Hypertension, a leading risk factor for global cardiovascular disease, contributes to half of coronary heart disease and approximately two-thirds of cerebrovascular disease burdens [1]. While the pathogenesis of hypertension remains ambiguous, hypertension is generally believed to be a complex and multifactorial disease. Recent

\footnotetext{
*Correspondence: fmulhy@163.com; fmuwsy@163.com

${ }^{\dagger}$ Equal contributors

${ }^{5}$ Department of Preventive Medicine, Fujian Provincial Key Laboratory of Environment Factors and Cancer, School of Public Health, Fujian Medical University, No. 1, Xueyuan Road, Fuzhou 350122, Fujian, China

'Department of Epidemiology and Health Statistics, School of Public Health, Fujian Medical University, No. 1, Xueyuan Road, Fuzhou 350122, Fujian, China Full list of author information is available at the end of the article
}

studies have revealed the relevance of noncoding RNAs with the pathogenesis of hypertension, supporting the importance of epigenomic regulation in hypertension progress $[2,3]$.

Currently, epigenetic regulators of hypertension mainly include long noncoding RNAs (lncRNAs), microRNAs (miRNAs) and DNA methylation [4]. LncRNAs have been recognised as critical regulators in the development of hypertension [5, 6]. Previous cell experiments have shown that NR_027032 regulates the function of vascular endothelial cells and is thought to be involved in vascular development, cell cycle, chromatin modification and DNA damage response genes [7]. NR_034083 is believed to regulate the proliferation, migration and 
phenotypic changes of vascular smooth muscle cells by regulating cell responses mediated by angiogenesis II [8]. NR_104181 has been shown to mediate rolling adhesion in $\mathrm{Ca}^{2+}$ and $\mathrm{Ca}^{2+}+\mathrm{Mg}^{2+}$ and firm adhesion in $\mathrm{Mg}^{2+}$ and $\mathrm{Mn}^{2+}$, mimicking the two key steps in leucocyte accumulation in the inflamed vasculature [9].

MiRNAs are involved in a variety of biological processes, including cellular proliferation, apoptosis and differentiation $[10,11]$. As the most highly enriched miRNA in endothelial cells, miR-126 can modulate human vascular endothelial phenotype in vitro and is required for the maintenance of vascular integrity and angiogenesis in vivo with a zebrafish model [12]. These findings were further confirmed by in vivo experiments with transgenic mice [10]. MiR-143 and miR-145 are cotranscribed in multipotent murine cardiac progenitors before becoming localised to smooth muscle cells and have been shown to cooperatively regulate the proliferation of vascular smooth muscle cells in transgenic mouse models [11, 13].

Despite the important role of noncoding RNAs (ncRNAs, including lncRNAs and miRNAs) in the development of hypertension, their relative contributions to hypertension remain largely unknown. Previous studies in humans have demonstrated that miRNAs (miR-126, miR-143 and miR-145) are differentially expressed in hypertensive patients and healthy individuals, though the expression levels of these miRNAs are not consistent among different reports [14-16]. There have been no previous studies on the expression levels of lncRNAs in hypertensive patient population samples. Thus, the goal of this study was to explore the relationship of cardiovascular disease risk factors and ncRNAs with essential hypertension $(\mathrm{EH})$ in the population and propose countermeasures for this disease.

\section{Methods}

\section{Study subjects}

From November 2014 to June 2016, we performed a hospital-based case-control study on EH in Fujian Province, China. Participants were recruited from the First Affiliated Hospital of Fujian Medical University and the Affiliated People's Hospital of Fujian University of Traditional Chinese Medicine. A total of $402 \mathrm{EH}$ patients were included in the final analysis. Meanwhile, 402 healthy controls were randomly selected from the physical examination population in the same hospitals, and were frequency-matched by gender and age $( \pm 3$ years $)$ with cases. The disease-free status was ascertained according to the results of physical examination. Furthermore, 80 of the $402 \mathrm{EH}$ patients and 80 of the 402 control individuals were selected by stratified random sampling method to donate venous blood samples for ncRNA expression analysis by quantitative real-time polymerase chain reaction (qPCR). Inclusion criteria for cases included patients with at least three consecutive records of systolic blood pressure $\geq 140 \mathrm{mmHg}$ and/or diastolic blood pressure $\geq 90 \mathrm{mmHg}$ or patients that have received antihypertensive medications for more than 3 months (European Society of HypertensionEuropean Society of Cardiology Guidelines, 2003). Inclusion criteria for controls included healthy individuals that have systolic blood pressure and diastolic blood pressure $<140$ and $<90 \mathrm{mmHg}$, respectively. Exclusion criteria included a known history of leucopenia, thrombocytopenia or severe hepatic or renal dysfunction and evidence of an inflammatory or malignant disease. The protocol was approved by the ethics committee of Fujian Medical University School. Written informed consent was obtained from all patients and controls.

\section{Questionnaire survey}

The participants completed the questionnaire themselves following instructions given by the researchers. The completed questionnaire was checked by the researchers to ensure that all questions have been answered appropriately. Questions covered demographic characteristics (e.g. gender, age, marital status, education level and occupation), lifestyle habits (e.g. tobacco smoking, alcohol drinking, diet, exercise, anxiety and depression levels) and family history of hypertension. Smokers were defined as individuals who smoked cigarettes more than once a day consecutively for more than 6 months or smoked more than 100 cigarettes cumulatively [17]. Alcohol drinkers were defined as subjects who had consumed any alcoholic beverage at least once per week for a minimum of 6 months [17]. A bland diet was defined as light food with no or very little oil, sugar, salt and spicy ingredients [17]. Occupational physical activities were classified into three categories depending on the intensity, duration and frequency of the participant's physical activity at work. Light occupational physical activity was defined as $75 \%$ of the time for sitting or standing and $25 \%$ of the time for standing activities, such as office workers, salesmen and hotel waiters; moderate occupational physical activity was defined as $25 \%$ of the time for sitting or standing and $75 \%$ of the time for special activities, such as students' daily activities, driving and electrical installation; and heavy occupational physical activity was defined as $40 \%$ of the time for sitting or standing and $60 \%$ of the time for special occupation activities, such as non-mechanised farming, dancing and mining [17]. The participants' personality was classified into four types: participants with type A personality were defined as those who were always in a rush, highly productive, impulsive, stubborn and impatient; participants with type B personality were defined as those who were always in a soothing and self- 
regulating state; participants with type $\mathrm{C}$ personality were defined as those who always suppressed their emotions and had too much negative emotional experience; and participants with type D personality were defined as those who had the tendency to experience increased negative emotions most of the time and not share these emotions with others because of fear of rejection or disapproval [17]. Active exercise was defined as exercise lasting for at least $20 \mathrm{~min}$ once a week, whereas passive exercise was defined as exercise lasting less than $20 \mathrm{~min}$ once a week or no exercise at all [17]. Anxiety and depression were self-rated according to a previous report [17]. The self-rating anxiety scale (SAS) was defined as follows: < 50 normal, 50-59 mild anxiety, 60-69 moderate anxiety and $\geq 70$ severe anxiety. The self-rating depression scale (SDS) was defined as follows: $<53$ normal, 53-62 mild anxiety, 63-73 moderate anxiety and $\geq 74$ severe anxiety.

\section{Physical examination}

Systolic and diastolic blood pressure, height, weight and waist circumference (WC) were measured. Before blood pressure measurement, each subject was asked to not smoke, drink any beverage with caffeine or exercise for at least half an hour. After resting in a sitting position for $5 \mathrm{~min}$, brachial blood pressure was measured three times with an interval of $2 \mathrm{~min}$; the average of three readings was used for analysis [17]. Body mass index (BMI) was calculated as weight $(\mathrm{kg})$ divided by the square of height in meters $\left(\mathrm{m}^{2}\right)$ and categorised into four scales: < 18.5 underweight, 18.5-24.0 normal, 24.028.0 overweight and $\geq 28.0$ obesity [17]. WC was classified into two categories: the waist of males $<85 \mathrm{~cm}$ or waist of females $<80 \mathrm{~cm}$ was considered normal, whereas waist of males $\geq 85 \mathrm{~cm}$ or waist of females $\geq 80 \mathrm{~cm}$ was considered abdominal obesity [17].

\section{Blood samples}

Blood samples were collected in a K2-EDTA-coated tube (BD Biosciences, California, USA) and centrifuged at $400 \mathrm{~g}$ for $10 \mathrm{~min}$ to remove plasma. According to the manufacturer's instructions for the Lymphocyte Separation Medium (Hao Yang Biological Manufacture Co., Ltd., Tianjin, China), blood samples were carefully transferred into a new RNA-free tube for a second centrifugation at $400 \mathrm{~g}$ for $20 \mathrm{~min}$ to obtain the peripheral blood leucocytes and then stored at $-80{ }^{\circ} \mathrm{C}$ prior to RNA extraction. All blood samples were subjected to only one freeze-thaw cycle.

\section{RNA extraction and qPCR}

Total RNA was extracted from peripheral blood leucocytes using TRIzol reagent (Invitrogen, California, USA) and dissolved in RNase-free water. RNA extracts were quantified using NanoDrop 2000 instrument (Thermo Scientific, USA). The integrity of the RNA was determined by agarose gel electrophoresis. Reverse transcription of quantified RNA was performed using PrimeScript RT Reagent Kit with gDNA Eraser (lncRNAs) or the PrimeScript RT Reagent Kit (miRNAs) (Takara Bio Inc., Shiga, Japan) according to the manufacturer's instructions. qPCR was performed on the LightCycler 480 real-time PCR system (Roche, Switzerland) with the SYBR ${ }^{\circ}$ Premix Ex Taq ${ }^{\text {tix }}$ II kit (Takara Bio Inc., Shiga, Japan). Amplification curves were obtained by 40 cycles of $95^{\circ} \mathrm{C}$ for $30 \mathrm{~s}, 95^{\circ} \mathrm{C}$ for $5 \mathrm{~s}$ and $60{ }^{\circ} \mathrm{C}$ for $34 \mathrm{~s}$, whereas dissolution curves were obtained by one cycle of $95{ }^{\circ} \mathrm{C}$ for $15 \mathrm{~s}, 60{ }^{\circ} \mathrm{C}$ for $1 \mathrm{~min}$ and $95{ }^{\circ} \mathrm{C}$ for $15 \mathrm{~s}$. The $\mathrm{Ct}$ value was the fractional cycle number at which the fluorescence exceeded the given threshold. GAPDH and U6 were used as internal controls. The reverse primer used for miRNA was a universal downstream primer. LncRNA and miRNA expression levels were calculated using the $2^{-\triangle \Delta C T}$ method [18]. The primers used for qPCR are listed in Table 1.

\section{Statistical analysis}

All data were analysed using SPSS 22.0 (SPSS Inc., Chicago, IL, USA). Comparisons between the case and control groups were performed using a Z-test or Chi-square analysis. Multiple factor logistic regression was used to analyse whether lncRNAs and miRNAs were influencing factors of EH. All tests were two sided and a $P$ value $<0.05$ was considered statistically significant.

\section{Results}

\section{Characteristics of the study population}

Associations between demographic characteristics and $\mathrm{EH}$ are shown in Table 2. No significant difference was found in the general demographic characteristics between the case and control groups, including gender, age, marital status, education and occupation (all $P>0.05$ ), indicating that the frequency matching was adequate. Associations between risk factors and $\mathrm{EH}$ are shown in Table 3. Compared with controls, EH patients had a lower bland diet rate $(61.2 \%$ versus $75.6 \%, X^{2}$ test, $\left.P<0.001\right)$, higher abdominal obesity rate $\left(84.0 \%\right.$ versus $64.7 \%, X^{2}$ test, $\left.P<0.001\right)$ and higher family history of hypertension rate $\left(47.0 \%\right.$ versus $19.5 \%, \chi^{2}$ test, $P<0.001$ ). Significant differences were found between cases and controls in the occupational physical activities, personality types, anxiety levels and body mass indices.

\section{LncRNAs and MiRNAs were differentially expressed in hypertensive patients and controls}

When lncRNA levels between hypertensive patients and controls were compared, we found that NR_027032 $(Z$-test $=-2.439, P=0.015)$ and NR_034083 $(Z$-test $=-2$. $890, P=0.004)$ were significantly reduced, whereas NR 104181 (Z-test $=-2.685, \quad P=0.007$ ) was significantly 
Table 1 List of the primers used for real time-PCR experiments

\begin{tabular}{|c|c|c|}
\hline Noncoding RNAs & Oligo Name & Sequence ( $5^{\prime}$ to $\left.3^{\prime}\right)$ \\
\hline \multirow[t]{2}{*}{ GAPDH } & Forward primer & GGACTCATGACCACAGTCCATGCC \\
\hline & Reverse primer & TCAGGGATGACCTTGCCCACAG \\
\hline \multirow[t]{2}{*}{ NR_027032 } & Forward primer & GTCCTCCACTCCACCTCAAA \\
\hline & Reverse primer & TGAGTTCCTGATCGTGTCCA \\
\hline \multirow[t]{2}{*}{ NR_034083 } & Forward primer & GGCTTCTAATCCGCCCTATC \\
\hline & Reverse primer & CAATGACCCAAGGCAAATTC \\
\hline \multirow[t]{2}{*}{ NR_104181 } & Forward primer & CCATTCAGCTTTCACCATGTGC \\
\hline & Reverse primer & ACCTTCAGGCGAGTCCAGATT \\
\hline \multirow[t]{2}{*}{ U6 } & Forward primer & GCTTCGGCAGCACATATACTAA \\
\hline & Reverse primer & AACGCTTCACGAATTTGCGT \\
\hline \multirow[t]{2}{*}{ miR-126 } & RT primer & GTCGTATCCAGTGCAGGGTCCGAGGTATTCGCACTGGATACGACCGCATT \\
\hline & Forward primer & GCGGCGGTCGTACCGTGAGTAA \\
\hline \multirow[t]{2}{*}{ miR-143 } & RT primer & GTCGTATCCAGTGCAGGGTCCGAGGTATTCGCACTGGATACGACGAGCTA \\
\hline & Forward primer & GCGGCGGTGAGATGAAGCACTG \\
\hline \multirow[t]{2}{*}{$\operatorname{miR}-145$} & RT primer & GTCGTATCCAGTGCAGGGTCCGAGGTATTCGCACTGGATACGACAGGGAT \\
\hline & Forward primer & GCGGCGGGTCCAGTTITCCCAG \\
\hline Universal downstream primer & Reverse primer & ATCCAGTGCAGGGTCCGAGG \\
\hline
\end{tabular}

elevated in hypertensive patients compared with controls (Table 4). When miRNA levels between hypertensive patients and controls were compared, we found that miR-143 (Z-test $=-2.797, P=0.005)$ and miR-145 (Ztest $=-2.436, P=0.015)$ were up-regulated in hypertensive patients compared with controls, whereas miR-126 level (Z-test $=-1.679, \quad P=0.093)$ was not significantly different between hypertensive patients and controls (Table 4).

\section{NR_034083 was a protective factor for EH whereas NR 104181 and miR-143 were risk factors}

We used $\mathrm{EH}$ as the dependent variable $(0=$ no, $1=$ yes $)$ and bland diet, occupation physical activities, personality

Table 2 Demographic characteristics of hypertensive patients and control subjects

\begin{tabular}{|c|c|c|c|c|c|}
\hline Characteristics & & $\begin{array}{l}\text { Hypertensive patients } \\
(n=402)\end{array}$ & Controls $(n=402)$ & $x^{2}$ & $P$ \\
\hline \multirow[t]{2}{*}{ Gender } & Male & $187(46.5)$ & $187(46.5)$ & 0.000 & 1.000 \\
\hline & Female & $215(53.5)$ & $215(53.5)$ & & \\
\hline \multirow[t]{3}{*}{ Age group (years) } & $<45$ & $22(5.5)$ & $22(5.5)$ & 0.000 & 1.000 \\
\hline & $45-65$ & $216(53.7)$ & $216(53.7)$ & & \\
\hline & $\geq 65$ & $164(40.8)$ & $164(40.8)$ & & \\
\hline \multirow[t]{3}{*}{ Marital status } & Single & $4(1.0)$ & $2(0.5)$ & 0.850 & 0.660 \\
\hline & Married & $363(90.5)$ & $369(91.8)$ & & \\
\hline & Divorce/widowed & $34(8.5)$ & $31(7.7)$ & & \\
\hline \multirow[t]{4}{*}{ Education level } & Elementary degree and below & $148(36.8)$ & $133(33.2)$ & 3.178 & 0.365 \\
\hline & Secondary degree & $101(25.1)$ & $123(30.7)$ & & \\
\hline & Senior/technical secondary degree & $104(25.9)$ & $99(24.7)$ & & \\
\hline & Associate degree and above & $49(12.2)$ & $46(11.5)$ & & \\
\hline \multirow[t]{4}{*}{ Occupation } & Worker & $69(17.3)$ & $77(19.2)$ & 0.646 & 0.886 \\
\hline & Farmer & $29(7.3)$ & $26(6.5)$ & & \\
\hline & Retired personnel & $181(45.3)$ & $177(44.0)$ & & \\
\hline & Other occupation & $121(30.3)$ & $122(30.3)$ & & \\
\hline
\end{tabular}


Table 3 Comparison of risk factors between hypertensive patients and healthy controls

\begin{tabular}{|c|c|c|c|c|c|}
\hline Risk factors & & Hypertensive patients $(n=402)$ & Controls $(n=402)$ & $x^{2}$ & $P$ \\
\hline \multirow[t]{2}{*}{ Smoking } & Yes & 87 (21.6) & $102(25.4)$ & 1.556 & 0.212 \\
\hline & No & $315(78.4)$ & $300(74.6)$ & & \\
\hline \multirow[t]{2}{*}{ Drinking } & Yes & $55(13.7)$ & $55(13.7)$ & 0.000 & 1.000 \\
\hline & No & $347(86.3)$ & $347(86.3)$ & & \\
\hline \multirow[t]{2}{*}{ Bland diet } & Yes & $246(61.2)$ & 304 (75.6) & 19.360 & $<0.001$ \\
\hline & No & $156(38.8)$ & $98(24.4)$ & & \\
\hline \multirow[t]{3}{*}{ Occupational physical activities } & Light & $187(46.5)$ & $143(35.6)$ & 10.451 & 0.005 \\
\hline & Moderate & $160(39.8)$ & $200(49.8)$ & & \\
\hline & Heavy & $55(13.7)$ & $59(14.7)$ & & \\
\hline \multirow[t]{3}{*}{ Exercise } & $<$ once/week & $211(52.5)$ & $197(49.0)$ & 3.157 & 0.206 \\
\hline & 1-2 times/week & $14(3.5)$ & $24(6.0)$ & & \\
\hline & $\geq 3$ times/week & $177(44.0)$ & $181(45.0)$ & & \\
\hline \multirow[t]{4}{*}{ Personality type } & A type & $139(34.6)$ & $88(21.9)$ & 17.436 & 0.001 \\
\hline & B type & $234(58.2)$ & $284(70.6)$ & & \\
\hline & C type & $22(5.5)$ & $26(6.5)$ & & \\
\hline & D type & $7(1.7)$ & $4(1.0)$ & & \\
\hline \multirow[t]{4}{*}{ Anxiety level } & Normal & $227(56.5)$ & $290(72.3)$ & 23.534 & $<0.001$ \\
\hline & Mild & $140(34.8)$ & $95(23.7)$ & & \\
\hline & Moderate & $31(7.7)$ & $15(3.7)$ & & \\
\hline & Severe & $4(1.0)$ & $1(0.2)$ & & \\
\hline \multirow[t]{4}{*}{ Depression level } & Normal & $241(60.0)$ & $251(62.4)$ & 1.670 & 0.684 \\
\hline & Mild & $119(29.6)$ & $104(25.9)$ & & \\
\hline & Moderate & $40(10.0)$ & $44(10.9)$ & & \\
\hline & Severe & $2(0.5)$ & $3(0.7)$ & & \\
\hline \multirow[t]{4}{*}{ Body mass index } & $<18.50$ & $8(2.0)$ & $23(5.7)$ & 46.103 & $<0.001$ \\
\hline & $18.50-23.99$ & $159(39.6)$ & $231(57.5)$ & & \\
\hline & $24.00-27.99$ & $177(44.0)$ & $127(31.6)$ & & \\
\hline & $\geq 28.00$ & $58(14.4)$ & $21(5.2)$ & & \\
\hline \multirow[t]{2}{*}{ Abdominal obesity } & Yes & $336(84.0)$ & $260(64.7)$ & 39.221 & $<0.001$ \\
\hline & No & $64(16.0)$ & $142(35.3)$ & & \\
\hline \multirow[t]{2}{*}{ Family history of hypertension } & Yes & $189(47.0)$ & $78(19.5)$ & 68.349 & $<0.001$ \\
\hline & No & $213(53.0)$ & $322(80.5)$ & & \\
\hline
\end{tabular}

Data are presented as $\mathrm{n}(\%)$

Table 4 Relative expression levels of IncRNAs and miRNAs in hypertensive patients and healthy controls $\left(P_{50}\left(P_{25}, P_{75}\right)\right)$

\begin{tabular}{lllrl}
\hline RNAs & Hypertensive patients $(n=80)$ & Controls $(n=80)$ & Z-test & $P$ \\
\hline NR_027032 & $0.839(0.402,1.485)$ & $1.312(0.493,1.923)$ & -2.439 & 0.015 \\
NR_034083 & $0.714(0.513,1.100)$ & $0.940(0.693,1.588)$ & -2.890 & 0.004 \\
NR_104181 & $1.946(0.880,3.013)$ & $1.253(0.568,2.275)$ & -2.685 & 0.007 \\
miR-126 & $1.751(0.854,2.904)$ & $1.229(0.548,2.340)$ & -1.679 & 0.093 \\
miR-143 & $1.796(0.644,3.470)$ & $1.013(0.420,1.984)$ & -2.797 & 0.005 \\
miR-145 & $1.708(0.758,2.945)$ & $1.022(0.585,2.064)$ & -2.436 & 0.015 \\
\hline
\end{tabular}


type, anxiety level, BMI, abdominal obesity and family history of hypertension as independent variables for multivariate logistic regression analysis (assignment results are shown in Table 5, wherein personality type and BMI were used as dummy variables). After adjusting the demographic and environmental factors, logistic analysis showed that lower expression levels of NR_034083 (OR $=0.528,95 \% C I=0.322-0.866)$ were a protective factor against hypertension, whereas higher expression levels of NR_104181 $(O R=1.651,95 \% C I=1.164-2.342)$ and miR-143 $(O R=1.538,95 \% C I=1.182-2.000)$ were risk factors (Table 6).

\section{Discussion}

In this case-control study, we first assessed the effects of common cardiovascular disease risk factors on hypertension in Fuzhou, China. Our study found that anxiety, BMI, abdominal obesity and family history of hypertension were risk factors for hypertension, whereas bland diet and occupational physical activities were protective factors for hypertension. Among the common risk factors, we found that higher levels of anxiety were associated with higher risk of hypertension. Anxiety may decrease the sensitivity of pressure reflex, leading to an increase of blood vessel tension and blood pressure as has been hypothesised previously [19].

In this study, we also assessed the association of six ncRNAs with hypertension and found that NR_027032 and NR_034083 were significantly reduced in hypertensive patients compared with control subjects. However, NR_104181, miR-143 and miR-145 were elevated in hypertensive patients compared with controls. After adjusting the demographic and environmental factors, multivariate analysis showed that lower expression levels of NR_034083 and higher expression levels of NR 104181 and miR-143 were risk factors for hypertension. In this study, the higher expression levels of miR-143 and miR-145 in hypertensive patients than in controls concur with previous reports from Paola Caruso et al. [14] and Santovito et al. [15] but are contradictory to the observations by Kontaraki et al. [16]. The absence of
Table 6 Multivariate logistic regression analysis of factors influencing the risk of hypertension

\begin{tabular}{llll}
\hline & $O R$ & $95 \%$ Cl of OR & $P$ value \\
\hline Anxiety & 2.737 & $1.344-5.574$ & 0.006 \\
Family history of hypertension & 3.842 & $1.142-12.929$ & 0.030 \\
NR_104181 & 1.651 & $1.164-2.342$ & 0.005 \\
NR_034083 & 0.528 & $0.322-0.866$ & 0.011 \\
miR-143 & 1.538 & $1.182-2.000$ & 0.001 \\
\hline
\end{tabular}

a significant difference in miR-126 expression levels between hypertensive patients and controls in our study is consistent with the report of Corsten et al. [20] but contrasts the report of Kontaraki et al. [21]. The reasons for these discrepancies are unclear but may be related to different blood samples (plasma [16], peripheral blood mononuclear cells [14, 21], peripheral blood leucocytes, peripheral total blood [20] and atherosclerotic plaques [15]), different disease processes (treated $[15,20]$ and untreated [16, 21] EH patients), different patient populations or undefined environmental factors. Clearly, further studies are needed to clarify these discrepancies.

Most importantly, we demonstrated for the first time that lncRNAs, similar to some miRNAs, are differentially expressed in hypertensive patients and healthy individuals, supporting their roles in the risk of human hypertension. The reduction of NR_034083 expression in hypertensive patients compared with controls suggests that this ncRNA is possibly a protective factor for hypertension. In support of this hypothesis, animal studies have demonstrated that NR_034083 is involved in negatively regulating angiotensin II [8], thereby leading to the development of hypertension. The up-regulation of NR 104181 expression in hypertensive patients suggests that this ncRNA is possibly a risk factor for hypertension, consistent with previous studies showing that NR 104181 can activate the function of lymphocytes, releasing a variety of inflammatory mediators to cause vascular dysfunction, including vasoconstriction, sodium and water retention and inhibiting the decrease of blood pressure $[9,22,23]$.

Table 5 Multivariate analysis variables and assignment

\begin{tabular}{lllll}
\hline Variables & Assignment & & & \\
\cline { 2 - 4 } & 0 & Yes & & \\
\hline Bland diet & No & Moderate & Heavy & \\
Occupational physical activities & Light & A type (1.0.0) & C type (0.1.0) & D type (0.0.1) \\
Personality type & B type (0.0.0) & Mild & Moderate & Severe \\
Anxiety level & Normal & $<18.50(1.0 .0)$ & 24.00-27.99 (0.1.0) & \\
Body mass index & $18.50-23.99(0.0 .0)$ & Yes & & \\
Abdominal obesity & No & Yes & & \\
Family history of hypertension & No & & & \\
\hline
\end{tabular}


Several limitations in our study need to be addressed. Firstly, recall bias was inevitable in this case-control study. However, it did not affect the ncRNA expression levels. Thus, it has a limited impact in studying epigeneticdisease association. Secondly, in the present study, blood samples collected can only present a single time point expression of lncRNAs or miRNAs. Thus, we did not look at the variability of the ncRNA expression level at different time points or under different conditions from the same individual [24]. Further mechanistic studies are needed to determine how these ncRNAs play their roles in hypertension. In addition, though the ncRNAs surveyed in this study, especially miR-126, miR-143 and miR-145, are known to be highly expressed in vascular endothelial and smooth muscle cells $[14,15]$, they might serve as attractive novel diagnostic biomarkers in peripheral blood leucocytes for human diseases.

\section{Conclusions}

Our findings support previous observations of the effects of common cardiovascular disease risk factors on hypertension. In addition, our study demonstrated for the first time that lncRNAs (NR_027032, NR_034083, NR_ 104181), similar to some miRNAs, are differentially expressed in hypertensive patients and healthy individuals, supporting their roles in the pathogenesis of EH.

\section{Abbreviations}

BMI: Body mass index; DBP: Diastolic blood pressure; EH: Essential hypertension; IncRNA: Long noncoding RNA; miRNA: MicroRNA; ncRNA: Noncoding RNA; SAS: Self-rating anxiety scale; SBP: Systolic blood pressure; SDS: Self-rating depression scale; WC: Waist circumference

\section{Acknowledgements}

The authors would like to thank the doctors and patients for their support and cooperation in sample collection and questionnaire completion.

\section{Funding}

This study was supported by the Training Project for Young- and MiddleAged Talents of the Fujian Province's Health System, China (grant No. 2014ZQN-ZD-24), the Local College and University Development Foundation of the Central Government (grant No. 1003-03900130-15), and the Natural Science Foundation of Fujian Province (grant No. 2015J01674).

\section{Availability of data and materials}

The datasets used and/or analysed during the current study are available from the corresponding author on reasonable request.

\section{Authors' contributions}

SYC and RC conceived the study and were involved in the experimental design, coordination and drafting the manuscript. TXZ, ZC and BZ participated in the data collection, result interpretation and manuscript writing. SWL performed the statistical analysis. HYL and SYW participated in study design and revising the manuscript. All authors read and approved the final manuscript.

\section{Ethics approval and consent to participate}

This study was approved by the ethics committee of Fujian Medical University School. Written informed consent was obtained from all patients and controls.

\section{Consent for publication}

Not applicable.

\section{Competing interests}

The authors declare that they have no competing interests.

\section{Publisher's Note}

Springer Nature remains neutral with regard to jurisdictional claims in published maps and institutional affiliations.

\section{Author details}

'Department of Epidemiology and Health Statistics, School of Public Health, Fujian Medical University, No. 1, Xueyuan Road, Fuzhou 350122, Fujian, China. ${ }^{2}$ Department of Cardiology, The First Affiliated Hospital of Fujian Medical University, Fuzhou 350005, Fujian, China. ${ }^{3}$ Department of Medical Management, Fujian University of Traditional Chinese Medicine Union People's Hospital, Fuzhou 350004, Fujian, China. ${ }^{4}$ Department of Nursing, Fuzhou General Hospital of Nanjing Military Command, Fuzhou 350001, Fujian, China. ${ }^{5}$ Department of Preventive Medicine, Fujian Provincial Key Laboratory of Environment Factors and Cancer, School of Public Health, Fujian Medical University, No. 1, Xueyuan Road, Fuzhou 350122, Fujian, China.

Received: 3 January 2018 Accepted: 20 March 2018

Published online: 02 April 2018

\section{References}

1. Whitworth JA. 2003 World Health Organization (WHO)/International Society of Hypertension (ISH) statement on management of hypertension. J Hypertens. 2003;21:1983-92.

2. Yao QP, Xie ZW, Wang KX, Zhang P, Han Y, Qi YX, et al. Profiles of long noncoding RNAs in hypertensive rats: long noncoding RNA XR007793 regulates cyclic strain-induced proliferation and migration of vascular smooth muscle cells. J Hypertens. 2017;35:1195-203.

3. Zhang R, Sui L, Hong X, Yang M, Li W. MiR-448 promotes vascular smooth muscle cell proliferation and migration in through directly targeting MEF2C. Environ Sci Pollut Res Int. 2017;24:22294-300.

4. Roosbroeck KV, Pollet J, Calin GA. miRNAs and long non-coding RNAs as biomarkers in human diseases. Expert Rev Mol Diagn. 2013;13:183-204.

5. Sun L, Goff LA, Trapnell C, Alexander R, Lo KA, Hacisuleyman E, et al. Long noncoding RNAs regulate adipogenesis. Proc Natl Acad Sci U S A. 2013;110:3387-92

6. Yu J, Cai Y, Liu Y, Yang Y, Chen Y, Jose PA, et al. 1A.07: genome-wide profiling of long noncoding Rna expression patterns in thoracic aorta from spontaneously hypertensive rats. J Hypertens. 2015;33(Suppl 1):e2.

7. Kurian L, Aguirre A, Sancho-Martinez I, Benner C, Hishida T, Nguyen TB, et al. Identification of novel long non-coding RNAs underlying vertebrate cardiovascular development. Circulation. 2015;131:1278-90.

8. Leung A, Trac C, Jin W, Lanting L, Akbany A, Sætrom P, et al. Novel long noncoding RNAs are regulated by angiotensin II in vascular smooth muscle cells. Circ Res. 2013;113:266-78.

9. Chen J, Salas A, Springer TA. Bistable regulation of integrin adhesiveness by a bipolar metalion cluster. Nat Struct Biol. 2003;10:995-1001.

10. Wang S, Aurora AB, Johnson BA, Qi X, McAnally J, Hill JA, et al. The endothelial-specific microRNA miR-126 governs vascular integrity and angiogenesis. Dev Cell. 2008;15:261-71.

11. Owens GK, Kumar MS, Wamhoff BR. Molecular regulation of vascular smooth muscle cell differentiation in development and disease. Physiol Rev. 2004;84:767-801.

12. Fish JE, Santoro MM, Morton SU, Yu S, Yeh RF, Wythe JD, et al. miR126 regulates angiogenic signaling and vascular integrity. Dev Cell. 2008;15:272-84

13. Cordes KR, Sheehy NT, White MP, Berry EC, Morton SU, Muth AN, et al. miR145 and miR-143 regulate smooth muscle cell fate and plasticity. Nature. 2009:460:705-10.

14. Caruso P, Dempsie Y, Stevens HC, McDonald RA, Long L, Lu R, et al. A role for miR-145 in pulmonary arterial hypertension: evidence from mouse models and patient samples. Circ Res. 2012;111:290-300.

15. Santovito D, Mandolini C, Marcantonio P, De Nardis V, Bucci M, Paganelli C, et al. Overexpression of microRNA-145 in atherosclerotic plaques from hypertensive patients. Expert Opin Ther Targets. 2013;17:217-23.

16. Kontaraki JE, Marketou ME, Zacharis EA, Parthenakis FI, Vardas PE. Differentia expression of vascular smooth muscle-modulating microRNAs in human 
peripheral blood mononuclear cells: novel targets in essential hypertension. J Hum Hypertens. 2014;28:510-6.

17. Liu LS. 2010 Chinese guidelines for the management of hypertension. Zhonghua Xin Xue Guan Bing Za Zhi. 2011;39:579-615.

18. Livak KJ, Schmittgen TD. Analysis of relative gene expression data using real-time quantitative PCR and the $2-\Delta \triangle C T$ method. Methods. 2001;25:402-8.

19. Wang DQ, Center CE, College JM. Effect of anxiety depression on ambulatory blood pressure in elderly hypertension patients. Chin J Geriatr Heart Brain Vessel Dis. 2014;16:723-5.

20. Corsten MF, Dennert R, Jochems S, Kuznetsova T, Devaux Y, Hofstra L, et al. Circulating MicroRNA-208b and MicroRNA-499 reflect myocardial damage in cardiovascular disease. Circ Cardiovasc Genet. 2010;3:499-506.

21. Kontaraki JE, Marketou ME, Zacharis EA, Parthenakis FI, Vardas PE. MicroRNA9 and microRNA-126 expression levels in patients with essential hypertension: potential markers of target-organ damage. J Am Soc Hypertens. 2014;8:368-75.

22. Harrison DG, Gongora MC. Oxidative stress and hypertension. Med Clin N Am. 2007;93:621-35.

23. Guzik TJ, Hoch NE, Brown KA, McCann LA, Rahman A, Dikalov S, et al. Role of the T cell in the genesis of angiotensin Ang II-induced hypertension and vascular dysfunction. J Exp Med. 2007;204:2449-60

24. Kane NM, Meloni M, Spencer HL, Craig MA, Strehl R, Milligan G, et al. Derivation of endothelial cells from human embryonic stem cells by directed differentiation: analysis of microRNA and angiogenesis in vitro and in vivo. Arterioscler Thromb Vasc Biol. 2010;30:1389-97.

\section{Submit your next manuscript to BioMed Central} and we will help you at every step:

- We accept pre-submission inquiries

- Our selector tool helps you to find the most relevant journal

- We provide round the clock customer support

- Convenient online submission

- Thorough peer review

- Inclusion in PubMed and all major indexing services

- Maximum visibility for your research

Submit your manuscript at www.biomedcentral.com/submit 